\title{
Synthesis of Formation Control Systems for Multi-Agent Systems under Control Gain Perturbations
}

\author{
Kazuki Miyakoshi ${ }^{1, *}$, Shun Ito ${ }^{1}$, Hidetoshi Oya ${ }^{1}$, Yoshikatsu Hoshi ${ }^{1}$, Shunya Nagai ${ }^{2}$ \\ ${ }^{1}$ Graduate School of Integrative Science and Engineering, Tokyo City University, Tokyo, Japan \\ ${ }^{2}$ Department of Information Systems Creation, Kanagawa University, Kanagawa, Japan \\ Received 25 April 2019; received in revised form 28 July 2019; accepted 26 November 2019
}

DOI: https://doi.org/10.46604/aiti.2020.4136

\begin{abstract}
This paper proposed a linear matrix inequality (LMI)-based design method of non-fragile guaranteed cost controllers for multi-agent systems (MASs) with leader-follower structures. In the guaranteed cost control approach, the resultant controller guarantees an upper bound on the given cost function together with asymptotical stability for the closed-loop system. The proposed non-fragile guaranteed cost control system can achieve consensus for MASs despite control gain perturbations. The goal is to develop an LMI-based sufficient condition for the existence of the proposed non-fragile guaranteed cost controller. Moreover, a design problem of an optimal non-fragile guaranteed cost controller showe that minimizing an upper bound on the given quadratic cost function can be reduced to constrain a convex optimization problem. Finally, numerical examples were given to illustrate the effectiveness of the proposed non-fragile controller for MASs.
\end{abstract}

Keywords: multi-agent systems (MASs), consensus, control gain perturbations, guaranteed cost control, LMIs

\section{Introduction}

The robustness of control systems is an important property, and thus "robust stability analysis" and "robust stabilization problems" have been well studied for a long time [1-2]. Especially, quadratic stabilizing controllers and control are typical robust control strategies [3-5]. Furthermore, for practical situations, it is desirable to design control systems that achieve not only asymptotical stability but also an adequate level of control performance. One approach to this problem is "the guaranteed cost control", and was introduced by Chang and Peng [6]. In the guaranteed cost control approach, a cost function corresponding to control performance introduced, and the resultant controller has the advantage of providing an upper bound on the given cost function. Namely, the system performance degradation incurred by the uncertainties is guaranteed to be less than this bound. Based on this idea, many significant results have been presented [7-9]. In the work of Petersen and McFarlane [7], the parameter-dependent Riccati equation approach adopted, and Yu and Chu have proposed a guaranteed cost controller design method based on linear matrix inequalities (LMIs) [8-9].

On the other hand, it is generally known that feedback systems designed for robustness concerning parameters in the controlled system may require very accurate controllers. However, uncertainties in controllers may appear for imprecision inherent in conversion and roundoff errors in numerical computations. Furthermore, it has pointed out that any useful design strategy should generate a controller which also has a sufficient margin for the readjustment of its coefficients [10-11]. In particular, Keel and Bhattacharyya [10] showed some examples that optimum and robust controllers can produce extremely fragile controllers, concerning vanishingly small perturbations for parameters of the designed controller. Namely, any useful design procedure should generate a controller which also has sufficient room for the readjustment of its coefficients because

\footnotetext{
* Corresponding author. E-mail address: g1881842@tcu.ac.jp
} 
controller implementation is subject to imprecision inherent in analog-digital and digital-analog conversion. Finite word length and finite resolution measuring instruments and roundoff errors in numerical computations. From this viewpoint, there are some efforts to tackle the design problem of robust non-fragile controllers [12-14]. Famularo et al. had shown a design method of a robust non-fragile LQ controller for linear systems with structured uncertainties in the system matrix [12]. Additionally, an LMI-based design method of the decentralized guaranteed cost controller for uncertain large-scale interconnected systems has been presented [13]. In the work of Oya et al. [14], for linear continuous-time systems with structured uncertainties included in the system matrix and the input under multiplicative or additive control gain variations, an LMI-based design method of a robust non-fragile stabilizing controller has been proposed.

By the way, with rapid technological development, controlled systems become large-scale and complex. One can see that systems are referred to as "large-scale interconnected systems". For large-scale interconnected systems, "centralized control strategies" might be unrealistic for physical, technical, and societal reasons. Also, one can see that "decentralized control strategies" are useful. Many researchers have studied decentralized control for large-scale interconnected systems (see [15] and references therein). For large-scale interconnected systems, some design methods for the decentralized robust control had been suggested [16-18]. Nagai et al. [17] proposed a decentralized variable to gain robust controller for a class of uncertain large-scale interconnected systems, and guaranteed cost control for large-scale interconnected systems with control gain perturbations has also been studied in [18]. Additionally, formation control for multi-agent systems (MASs) is known as one of the decentralized control problems and has recently attracted much attention. In general, a multi-agent system is a loosely coupled network of multiple interacting agents, and it is well-known that MASs can achieve various tasks efficiently. Although there are various topics in the formation control problem for MASs, the consensus problem has been well focused. The consensus problem can be applied to various fields such as sensor networks, vehicle formations, mobile robots, unmanned aerial vehicles, and so on. Additionally, consensus means that states of all agents are driven to a common state by implementing distributed protocols. Therefore, the consensus problems are one of the most important and fundamental in the formation, and lots of existing results for consensus problems have been shown (e.g. [19-22]). The consensus problem for a network of first-order integrators with directed in the formation flow and fixed/switching topology has been studied in [19]. Xie and Wang have studied convergence analysis of a consensus protocol for a class of networks of dynamic agents with fixed topology. Furthermore, Zhai et al. proposed the matrix inequality-based stabilization condition and consensus algorithm for MASs had been presented [22]. Ito et al. [23] proposed an adaptive gain controller design method considering relative distances for MASs. Although the existing result [23] had dealt with consensus problems for multi-agent systems with a leader-follower structure, control gain perturbations have not been considered. About these results of the consensus problem for MASs, uncertainties in controllers were not considered, and the LMI-based design method of non-fragile guaranteed cost controllers for MASs with control gain perturbations has little been considered as far as known.

From this viewpoint, a consensus problem for MASs with leader-follower structures was discussed, and an LMI-based design method of a non-fragile guaranteed cost controller which was presented guarantees a consensus for MASs. In this paper, additive control gain perturbations are dealt with and showed that sufficient conditions for the existence of the proposed non-fragile guaranteed cost controller given in terms of linear matrix inequalities (LMIs). Additionally, a design method of quadratic guaranteed cost control-based consensus strategy which guarantees the upper bound of a given quadratic cost function is considered. Moreover, the optimal guaranteed cost control approach which minimizes the upper bound of the quadratic cost function is discussed. The crucial differences between the proposed controller synthesis and the existing results [19-23] are that the proposed controller perturbations and optimal guaranteed cost control-based consensus strategy can be achieved. Besides, the proposed non-fragile controller can easily be obtained by solving the constrained convex optimization problem, i.e. the proposed non-fragile guaranteed cost control for a consensus is useful. Finally, a numerical example is presented to illustrate the effectiveness of the proposed guaranteed cost controller for formation control systems. 
This paper is organized as follows. In Section 2, notation and two useful lemmas which were used in this paper are shown. In Section 3, the main results presented. Namely, an LMI-based design method of a guaranteed cost controller for MASs under control gain perturbations. Additionally, the optimal guaranteed cost controller which was discussed minimizes the upper bound on a given quadratic cost function. Finally, simple illustrative examples were displayed to show the effectiveness of the non-fragile guaranteed cost controller developed in this paper.

\section{Notations and Lemmas}

The following notations were used in this paper. For a matrix $A$, the inverse matrix $A$ and its transpose are denoted by $A^{-1}$ and $A^{T}$, respectively. Also, $\operatorname{He}\{A\}$ means $A+A^{T}$ and $I_{n}$ represent $n$-dimensional identity matrix, and a block diagonal matrix is composed of matrices $A_{i}$ for $i=1,2, \cdots, M$ are described as $\operatorname{diag}\left\{A_{1}, A_{2}, \cdots, A_{M}\right\}$. Moreover, real symmetric matrix $A, A>0$ (resp. $A \geq 0$ ) means that $A$ is positive (resp. nonnegative) definite matrix. For a vector $a$, $\|a\|$ is Euclidian norm, and $\|A\|$ represents its included norm for a matrix $A$. The symbols “*” and " $\triangleq$ " mean symmetric blocks in matrix inequalities and equality by definition, respectively. Moreover, the Kronecker product of matrices $A \in \mathbb{R}^{m \times n}$ and $B \in \mathbb{R}^{p \times q}$ is defined as

$$
A \otimes B=\left(\begin{array}{ccc}
a_{11} B & \ldots & a_{1 n} B \\
\vdots & \ddots & \vdots \\
a_{m 1} B & \cdots & a_{m n} B
\end{array}\right)
$$

Furthermore, the following two useful lemmas were used in this paper:

Lemma 1 (Schur complement formula [24]): For a given constant real symmetric matrix $\psi$, the following items are equivalent:

(i) $\psi=\left(\begin{array}{cc}\psi_{11} & \psi_{12} \\ * & \psi_{22}\end{array}\right)>0$,

(ii) $\psi_{11}>0$ and $\psi_{22}-\psi_{12}^{T} \psi_{11}^{-1} \psi_{12}>0$,

(iii) $\psi_{22}>0$ and $\psi_{11}-\psi_{12} \psi_{22}^{-1} \psi_{12}^{T}>0$.

Lemma 2 [14]: For matrices $P$ and $H$, which have appropriate dimensions and a positive scalar $\gamma$, the following relation holds:

$$
P H+H^{T} P^{T} \leq \gamma P P^{T}+\frac{1}{\gamma} H^{T} H
$$

\section{Problem Formulation}

The multi-agent system which is considered is composed of 3 agents where an agent indexed by 1 acts as the leader and the other agents indexed by 2, 3, respectively, act as the followers described as the following state equation:

$$
\frac{d}{d t} x_{i}(t)=A x_{i}(t)+B u_{i}(t)
$$

where $x_{i}^{T}(t)=\left(x_{i}^{(1)}(t), x_{i}^{(2)}(t), x_{i}^{(3)}(t), x_{i}^{(4)}(t)\right)$ is the state of $i$-th agent and elements, $x_{i}^{(1)}(t)$ and $x_{i}^{(2)}(t)\left(\right.$ resp. $x_{i}^{(3)}(t)$ and $\left.x_{i}^{(4)}(t)\right)$ mean the position and velocity on $x$ axis (resp. $y$ axis), $A \in \mathbb{R}^{4 \times 4}$ and $B \in \mathbb{R}^{4 \times 2}$ are given by 


$$
A=\left(\begin{array}{llll}
0 & 1 & 0 & 0 \\
0 & 0 & 0 & 0 \\
0 & 0 & 0 & 1 \\
0 & 0 & 0 & 0
\end{array}\right), \quad B=\left(\begin{array}{ll}
0 & 0 \\
1 & 0 \\
0 & 0 \\
0 & 1
\end{array}\right)
$$

one can see that the overall system can be written as

$$
\frac{d}{d t} x(t)=A_{t} x(t)+B_{t} u(t)
$$

where $A_{t}=\operatorname{diag}\{A, A, A\}$ and $B_{t}=\operatorname{diag}\{B, B, B\}$. Moreover, $x(t)=\left(x_{l}^{T}(t) \quad x_{2}^{T}(t) \quad x_{3}^{T}(t)\right)^{T}$ and $u(t)=\left(u_{l}^{T}(t) \quad u_{2}^{T}(t) \quad u_{3}^{T}(t)\right)^{T} \quad$ in Eq. (5) are the vectors of the state and the control input for the overall system, respectively. It is well-known that the information path between agents based on graph theory, and the topology connection which is considered is shown in Fig. 1.

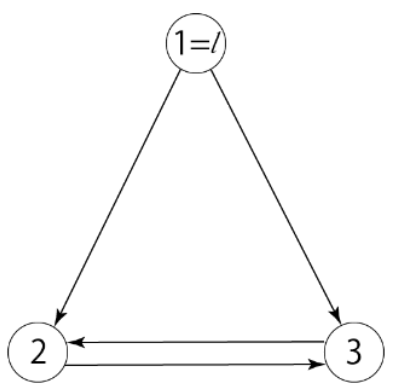

Fig. 1 The topology connection of agents

Since the topology connection can also be expressed by graph Laplacian $L$ defined by the adjacency matrix $A$ and the degree matrix $D$. In the topology connection, the adjacency matrix $A$ and the degree matrix $D$ are given by

$$
A=\left(\begin{array}{lll}
0 & 0 & 0 \\
1 & 0 & 1 \\
1 & 1 & 0
\end{array}\right), \quad D=\left(\begin{array}{lll}
0 & 0 & 0 \\
0 & 2 & 0 \\
0 & 0 & 2
\end{array}\right)
$$

Thus, the corresponding graph Laplacian $L$ can be described as

$$
L \triangleq D-A=\left(\begin{array}{ccc}
0 & 0 & 0 \\
-1 & 2 & -1 \\
-1 & -1 & 2
\end{array}\right)
$$

Now, to consider the control gain perturbations for the $i$-th agent of Eq. (3), there are the following control input:

$$
\begin{aligned}
u_{i}(t) & \triangleq u_{K, i}(t)+u_{F, i}(t) \\
& =K(t) x_{i}(t)+F(t) \sum_{j \in N_{i}}\left(x_{i}(t)-x_{j}(t)\right)
\end{aligned}
$$

where $N_{i}$ is the set of neighbors for the $i$-th agent. $K(t) \in \mathbb{R}^{2 \times 4}$ is the state feedback gain matrix and $F(t) \in \mathbb{R}^{2 \times 4}$ is the consensus gain matrix. These gain matrices are defined as

$$
\begin{aligned}
& K(t) \triangleq K+\Delta K(t) \\
& F(t) \triangleq F+\Delta F(t)
\end{aligned}
$$

Note that $K \in \mathbb{R}^{2 \times 4}$ and $F \in \mathbb{R}^{2 \times 4}$ are the nominal control gain matrices, and the matrices $\Delta K(t) \in \mathbb{R}^{2 \times 4}$ and $\Delta F(t) \in \mathbb{R}^{2 \times 4}$ are unknown time-varying parameters which satisfy the relations $\|\Delta K(t)\| \leq \varepsilon_{K}$ and $\|\Delta F(t)\| \leq \varepsilon_{F}$. One can see that additive control gain perturbations were considered in this paper. Additionally, $\varepsilon_{K} \in \mathbb{R}^{1}$ and $\varepsilon_{F} \in \mathbb{R}^{1}$ mean upper bounds of the 
control gain perturbations and are known positive scalars. Namely, for the overall system of Eq. (5) the actual control input implemented is assumed to be

$$
\begin{aligned}
u(t) & \triangleq u_{K}(t)+u_{F}(t) \\
& =K_{t}(t) x(t)+F_{t}(t) x(t)
\end{aligned}
$$

where $u_{p}(t)=\left(u_{p l}^{T}(t) \quad u_{p 2}^{T}(t) \quad u_{p 3}^{T}(t)\right)^{T}(p=K, F)$. In Eq. (11), $u_{K}(t) \in \mathbb{R}^{6}$ can be described as

$$
u_{K}(t) \triangleq\left(I_{3} \otimes K(t)\right) x(t)=K_{t}(t) x(t)
$$

and $u_{F}(t) \in \mathbb{R}^{6}$ is the consensus protocol given by

$$
\begin{aligned}
u_{F}(t) & =\left(\begin{array}{ccc}
0 & 0 & 0 \\
-F(t) & 2 F(t) & -F(t) \\
-F(t) & -F(t) & 2 F(t)
\end{array}\right)\left(\begin{array}{c}
x_{l}(t) \\
x_{2}(t) \\
x_{3}(t)
\end{array}\right) \\
& =(L \otimes F(t)) x(t) \\
& =F_{t}(t) x(t)
\end{aligned}
$$

Therefore, the actual control input of Eq. (11) can be rewritten as

$$
u(t)=\left(\begin{array}{ccc}
K(t) & 0 & 0 \\
-F(t) & K(t)+2 F(t) & -F(t) \\
-F(t) & -F(t) & K(t)+2 F(t)
\end{array}\right) x(t)
$$

Moreover, the closed-loop system found is described as

$$
\begin{aligned}
\frac{d}{d t} x(t) & =A_{t} x(t)+B_{t}\left(K_{t}(t) x(t)+F_{t}(t) x(t)\right) \\
& =\left(\left(\begin{array}{ccc}
A & 0 & 0 \\
* & A & 0 \\
* & * & A
\end{array}\right)+\left(\begin{array}{ccc}
B & 0 & 0 \\
* & B & 0 \\
* & * & B
\end{array}\right)\left(\begin{array}{ccc}
K(t) & 0 & 0 \\
-F(t) & K(t)+2 F(t) & -F(t) \\
-F(t) & -F(t) & K(t)+2 F(t)
\end{array}\right)\right)\left(\begin{array}{l}
x_{l}(t) \\
x_{2}(t) \\
x_{3}(t)
\end{array}\right) \\
& =\left(\left(\begin{array}{ccc}
A_{K} & 0 & 0 \\
-B F & A_{F} & -B F \\
-B F & -B F & A_{F}
\end{array}\right)+B_{t}\left(\Delta K_{t}(t)+\Delta F_{t}(t)\right)\left(\begin{array}{l}
x_{l}(t) \\
x_{2}(t) \\
x_{3}(t)
\end{array}\right)\right.
\end{aligned}
$$

where $A_{K} \in \mathbb{R}^{4 \times 4}$ and $A_{F} \in \mathbb{R}^{4 \times 4}$ are given by $A_{K}=A+B K$ and $A_{F}=A+B K+2 B F$, respectively. Also, $\Delta K_{t}(t)$ and $\Delta F_{t}(t)$ are unknown matrices which can be written as

$$
\Delta K_{t}(t)=\left(\begin{array}{ccc}
\Delta K(t) & 0 & 0 \\
* & \Delta K(t) & 0 \\
* & * & \Delta K(t)
\end{array}\right), \quad \Delta F_{t}(t)=\left(\begin{array}{ccc}
0 & 0 & 0 \\
-\Delta F(t) & 2 \Delta F(t) & -\Delta F(t) \\
-\Delta F(t) & -\Delta F(t) & 2 \Delta F(t)
\end{array}\right)
$$

Now, the following quadratic cost function introduced:

$$
J=\int_{0}^{\infty}\left(x^{T}(t) Q_{t} x(t)+u_{K}^{T}(t) R_{K_{t}} u_{K}(t)+u_{F}^{T}(t) R_{F_{t}} u_{F}(t)\right) d t
$$

where $Q_{t} \in \mathbb{R}^{12 \times 12}, R_{K_{t}} \in \mathbb{R}^{6 \times 6}$, and $R_{F_{t}} \in \mathbb{R}^{6 \times 6}$ are given positive-definite symmetric matrices that are selected by designers.

From the above discussion, the control objective in this paper is to design a guaranteed cost controller which minimizes the upper bound on the quadratic cost function of Eq. (17). That is to derive gain matrices $K \in \mathbb{R}^{2 \times 4}$ and $F \in \mathbb{R}^{2 \times 4}$ minimizing the upper bound on the quadratic cost function of Eq. (17). 


\section{Main Results}

In this section, a design method of the non-fragile controller showed based on the linear matrix inequality (LMI) framework.

Firstly, the following theorem is given for the non-fragile controller under additive control to gain perturbations of Eq. (11).

Theorem 1: For the overall system of Eq. (15) with the control input of Eq. (11) and the quadratic cost function of Eq. (17), if there are symmetric positive definite matrix $S>0$, matrices $W_{K}$ and $W_{F}$, and scalars $\gamma>0, \delta>0, \zeta>0$ and $\eta>0$ satisfying the following LMI condition:

$$
\begin{aligned}
& \left(\begin{array}{cccccccc}
\Gamma\left(S, W_{K}, W_{F}, \gamma, \delta\right) & S_{t} & S_{t} & S_{t} & W_{K_{t}}^{T} & W_{F_{t}}^{T} & S_{t} & S_{t} \\
S_{t} & -\frac{\gamma}{\varepsilon_{K}^{2}} I_{12} & 0 & 0 & 0 & 0 & 0 & 0 \\
S_{t} & 0 & -\frac{\delta}{5 \varepsilon_{F}^{2}} I_{12} & 0 & 0 & 0 & 0 & 0 \\
S_{t} & 0 & 0 & -Q_{t}^{-1} & 0 & 0 & 0 & 0 \\
W_{K_{t}} & 0 & 0 & 0 & -R_{K_{t}}^{-1}+\zeta \varepsilon_{K}^{2} I_{6} & 0 & 0 & 0 \\
W_{F_{t}} & 0 & 0 & 0 & 0 & -R_{F_{t}}^{-1}+6 \eta \varepsilon_{F}^{2} I_{6} & 0 & 0 \\
S_{t} & 0 & 0 & 0 & 0 & 0 & -\zeta I_{12} & 0 \\
S_{t} & 0 & 0 & 0 & 0 & 0 & 0 & -\eta I_{12}
\end{array}\right)<0 \\
& \Gamma\left(S, W_{K}, W_{F}, \gamma, \delta\right) \triangleq\left(\begin{array}{ccc}
\Gamma_{11}\left(S, W_{K}\right) & \Gamma_{12}\left(W_{F}\right) & \Gamma_{13}\left(W_{F}\right) \\
* & \Gamma_{22}\left(S, W_{K}, W_{F}, \gamma, \delta\right) & \Gamma_{23}\left(W_{F}\right) \\
* & * & \Gamma_{33}\left(S, W_{K}, W_{F}, \gamma, \delta\right)
\end{array}\right) \\
& \Gamma_{11}\left(S, W_{K}\right)=H e\left\{A S+B W_{K}\right\}, \Gamma_{12}\left(W_{F}\right)=\Gamma_{13}\left(W_{F}\right)=-W_{F}^{T} B^{T}, \Gamma_{23}\left(W_{F}\right)=H e\left\{-B W_{F}\right\} \\
& \Gamma_{22}\left(S, W_{K}, W_{F}, \gamma, \delta\right)=\Gamma_{33}\left(S, W_{K}, W_{F}, \gamma, \delta\right)=H e\left\{A S+B W_{K}+2 B W_{F}\right\}+(\gamma+\delta) B B^{T}
\end{aligned}
$$

then the upper bound on the quadratic cost function of Eq. (17) is guaranteed and the closed-loop system of Eq. (15) with gain matrices $K=W_{K} S^{-1}$ and $F=W_{F} S^{-1}$ are asymptotically stable. Moreover, the upper bound on the quadratic cost function of Eq. (17) is given by $J^{*}(x(0)) \triangleq x^{T}(0) P x(0)$.

Proof: Using a symmetric positive definite matrix $P \in \mathbb{R}^{4 \times 4}$, the following quadratic function introduced as a Lyapunov function candidate:

$$
V(x, t)=x(t)^{T}\left(I_{3} \otimes P\right) x(t)
$$

From Eqs. (5), (11), and (15), the time derivative of the quadratic function $V(x, t)$ which is along the trajectory of the closed-loop system of Eq. (15) can be computed as

$$
\begin{aligned}
\frac{d}{d t} V(x, t) & =\left(\frac{d}{d t} x^{T}(t)\right)\left(I_{3} \otimes P\right) x(t)+x^{T}(t)\left(I_{3} \otimes P\right)\left(\frac{d}{d t} x(t)\right) \\
& =x^{T}(t) H e\left[\left(I_{3} \otimes P\right)\left\{\Phi(K, F)+B_{t}\left(\Delta K_{t}(t)+\Delta F_{t}(t)\right)\right\}\right] x(t)
\end{aligned}
$$

where $\Phi(K, F) \in \mathbb{R}^{12 \times 12}$ is the matrix described as

$$
\Phi(K, F) \triangleq\left(\begin{array}{ccc}
A_{K} & 0 & 0 \\
-B F & A_{F} & -B F \\
-B F & -B F & A_{F}
\end{array}\right)
$$


Therefore, if there exists the state feedback gain matrix $K \in \mathbb{R}^{2 \times 4}$, consensus gain matrix $F \in \mathbb{R}^{2 \times 4}$ and the symmetric positive definite matrix $P \in \mathbb{R}^{4 \times 4}$ which satisfy the inequality condition

$$
H e\left[\left(I_{3} \otimes P\right)\left\{\Phi(K, F)+B_{t}\left(\Delta K_{t}(t)+\Delta F_{t}(t)\right)\right\}\right]<0
$$

then the quadratic function $V(x, t)$ satisfies the following inequality:

$$
\frac{d}{d t} V(x, t)<0, \forall x(t) \neq 0
$$

i.e. the quadratic function $V(x, t)$ becomes a Lyapunov function for the closed-loop system Eq. (15).

By considering the quadratic cost function of Eq. (17), there is an inequality condition:

$$
\Phi(K, F)+H e\left\{P_{t} B_{t}\left(\Delta K_{t}(t)+\Delta F_{t}(t)\right)\right\}+Q_{t}+K_{t}^{T}(t) R_{K_{t}} K_{t}(t)+F_{t}^{T}(t) R_{F_{t}} F_{t}(t)<0
$$

instead of the matrix inequality of Eq. (25). The matrix $S \triangleq P^{-1}$ introduced, and the change of variables $W_{K} \triangleq K S$ and $W_{F} \triangleq F S$ considered. Then pre- and post-multiplying Eq. (25) by $S \in \mathbb{R}^{4 \times 4}$ and using Lemma 2, there is

$$
\begin{aligned}
& \Psi\left(S, W_{K}, W_{F}\right)+(\gamma+\delta) B_{t} B_{t}^{T}+\frac{\varepsilon_{K}^{2}}{\gamma} S_{t} S_{t}+\frac{5 \varepsilon_{F}^{2}}{\delta} S_{t} S_{t}+S_{t} Q_{t} S_{t} \\
& +\left(W_{K_{t}}+\Delta K(t) S_{t}\right)^{T} R_{K_{t}}\left(W_{K_{t}}+\Delta K(t) S_{t}\right)+\left(W_{F_{t}}+\Delta F(t) S_{t}\right)^{T} R_{F_{t}}\left(W_{F_{t}}+\Delta F(t) S_{t}\right)<0
\end{aligned}
$$

where $\Psi\left(S, W_{K}, W_{F}\right) \in \mathbb{R}^{12 \times 12}$ is given by

$$
\begin{aligned}
& \Psi\left(S, W_{K}, W_{F}\right)=\left(I_{3} \otimes S\right) \Phi(P, K, F)\left(I_{3} \otimes S\right)=\left(\begin{array}{ccc}
\Phi_{11}\left(S, W_{K}\right) & \Phi_{12}\left(W_{F}\right) & \Phi_{13}\left(W_{F}\right) \\
* & \Phi_{22}\left(S, W_{K}, W_{F}\right) & \Phi_{23}\left(W_{F}\right) \\
* & * & \Phi_{33}\left(S, W_{K}, W_{F}\right)
\end{array}\right) \\
& \Psi_{11}\left(S, W_{K}\right)=H e\left\{A S+B W_{K}\right\}, \Psi_{12}\left(W_{F}\right)=\Psi_{13}\left(W_{F}\right)=-W_{F}^{T} B^{T}, \Psi_{23}\left(W_{F}\right)=H e\left\{-B W_{F}\right\} \\
& \Psi_{22}\left(S, W_{K}, W_{F}\right)=\Psi_{33}\left(S, W_{K}, W_{F}\right)=H e\left\{A S+B W_{K}+2 B W_{F}\right\}
\end{aligned}
$$

One can see that $\Gamma\left(S, W_{K}, W_{F}, \gamma, \delta\right) \in \mathbb{R}^{12 \times 12}$ can be expressed as

$$
\Gamma\left(S, W_{K}, W_{F}, \gamma, \delta\right) \triangleq \Psi\left(S, W_{K}, W_{F}\right)+(\gamma+\delta) B_{t} B_{t}^{T}
$$

Therefore, applying Lemma 1 to the inequality Eq. (28) and simple algebraic gives

$$
\left(\begin{array}{cccccc}
\Gamma\left(S, W_{K}, W_{F}, \gamma, \delta\right) & S_{t} & S_{t} & S_{t} & W_{K_{t}}^{T} & W_{F_{t}}^{T} \\
S_{t} & -\frac{\gamma}{\varepsilon_{K}^{2}} I_{12} & 0 & 0 & 0 & 0 \\
S_{t} & 0 & -\frac{\delta}{5 \varepsilon_{F}^{2}} I_{12} & 0 & 0 & 0 \\
S_{t} & 0 & 0 & -Q_{t}^{-1} & 0 & 0 \\
W_{K_{t}} & 0 & 0 & 0 & -R_{K_{t}}^{-1} & 0 \\
W_{F_{t}} & 0 & 0 & 0 & 0 & -R_{F_{t}}^{-1}
\end{array}\right)+\Omega(\Delta K, \Delta F)<0
$$

where $\Omega(\Delta K, \Delta F) \in \mathbb{R}^{12 \times 12}$ is given by 


$$
\Omega(\Delta K, \Delta F) \triangleq\left(\begin{array}{c}
0 \\
0 \\
0 \\
0 \\
\Delta K_{t}^{T}(t) \\
0
\end{array}\right)\left(\begin{array}{c}
S_{t} \\
0 \\
0 \\
0 \\
0 \\
0
\end{array}\right)^{T}+\left(\begin{array}{c}
S_{t} \\
0 \\
0 \\
0 \\
0 \\
0
\end{array}\right)\left(\begin{array}{c}
0 \\
0 \\
0 \\
0 \\
\Delta K_{t}^{T}(t) \\
0
\end{array}\right)^{T}+\left(\begin{array}{c}
0 \\
0 \\
0 \\
0 \\
0 \\
\Delta F_{t}^{T}(t)
\end{array}\right)\left(\begin{array}{c}
S_{t} \\
0 \\
0 \\
0 \\
0 \\
0
\end{array}\right)^{T}+\left(\begin{array}{c}
S_{t} \\
0 \\
0 \\
0 \\
0 \\
0
\end{array}\right)\left(\begin{array}{c}
0 \\
0 \\
0 \\
0 \\
0 \\
\Delta F_{t}^{T}(t)
\end{array}\right)^{T}
$$

Thus, one can see from Lemma 2 that the following inequality can be obtained:

$$
\left(\begin{array}{cccccc}
\Gamma\left(S, W_{K}, W_{F}, \gamma, \delta\right)+\left(\zeta^{-1}+\eta^{-1}\right) S_{t} S_{t} & S_{t} & S_{t} & S_{t} & W_{K_{t}}^{T} & W_{F_{t}}^{T} \\
S_{t} & -\frac{\gamma}{\varepsilon_{K}^{2}} I_{12} & 0 & 0 & 0 & 0 \\
S_{t} & 0 & -\frac{\delta}{5 \varepsilon_{F}^{2}} I_{12} & 0 & 0 & 0 \\
S_{t} & 0 & 0 & -Q_{t}^{-1} & 0 & 0 \\
W_{K_{t}} & 0 & 0 & 0 & -R_{K_{t}}^{-1}+\zeta \varepsilon_{K}^{2} I_{6} & 0 \\
W_{F_{t}} & 0 & 0 & 0 & 0 & -R_{F_{t}}^{-1}+6 \eta \varepsilon_{F}^{2} I_{6}
\end{array}\right)<0
$$

Furthermore, by applying Lemma 1 to the inequality of Eq. (35), the inequality of Eq. (35) which was found is equivalent to the LMI of Eq. (18). It follows that the result of the theorem is true. Therefore, the proof of Theorem 1 is completed. (Q.E.D)

Theorem 1 provides a sufficient condition to gain perturbations of the form of Eq. (15) for the existence of the guaranteed cost controller which is under control. Next, the theorem for the optimal guaranteed cost controller is showed. The LMI of Eq. (18) defines a convex solution set, and thus various efficient convex optimization algorithms can be adapted to test whether the LMIs are solvable and whether they generate particular solutions. Moreover, the parametrized representation exploited to design the guaranteed cost controller with some additional requirements, its solutions parametrize the set of guaranteed cost controllers. In particular, the optimal guaranteed cost controller which minimizes the upper bound on the quadratic cost function of Eq. (17) can be obtained by solving a certain optimization problem. The upper bound on the quadratic cost function of Eq. (17) depends on the initial value of the overall system. Thus, the complementary variable $\sigma$ which was introduced satisfies the following LMI:

$$
\left(\begin{array}{cc}
-\sigma & x^{T}(0) \\
x(0) & -S
\end{array}\right)<0
$$

One can easily see that the minimization of $\sigma$ is equivalent to the minimization of the upper bound on the quadratic cost function of Eq. (17). Consequently, the following theorem developed:

Theorem 2: Consider the overall system of Eq. (5) with control gain perturbations, the quadratic cost function of Eq. (17) and the control input of Eq. (11).

If there exists solutions of the following constrained convex optimization problem:

$$
S>0, W_{K}, W_{F}, \gamma>0, \delta>0, \zeta>0, \eta>0, \sigma>0 \text { Minimize }[\sigma] \text { subject to eqs.(18) and (34) }
$$

then the control input of Eq. (11) is an optimal guaranteed cost control which minimizes the upper bound on the quadratic cost function of Eq. (17).

Proof: Since the minimization of $\sigma$ is equivalent to the minimization of the upper bound on the quadratic cost function of Eq. (17), the result of Theorem 2 is obtained by adopting a similar way to the proof of Theorem 1 and the existing results (e.g. $[13-14,25])$. 
Remark 1: In this paper, a design problem of non-fragile guaranteed cost controllers which achieve consensus for multiagent systems (MASs) with leader-follower structures was studied, and for simplicity MASs composed of 3 agents had been considered. Additionally, LMI-based design for optimal non-fragile guaranteed cost control had also been discussed. One can easily see that the proposed controller design approach applies to MASs without leaders, and the proposed non-fragile controller can be extended to MASs composed of more than 3 agents.

\section{Numerical Examples}

To demonstrate the efficiency of the proposed non-fragile stabilizing controller, a simple example had been run. In this example, the non-fragile controller under the control gain perturbations is considered. Also, the simulation results are shown for the proposed robust stabilizing controller designed without thinking of control gain perturbations. The control problem considered here is not necessarily practical. However, the simulation results stated below illustrate the distinct feature of the proposed non-fragile controller, i.e. The proposed non-fragile controller was compared with the conventional consensus controller which was designed by ignoring controller gain perturbations.

Consider the multi-agent system of Eq. (5). In this example, $\varepsilon_{K}=3.5 \times 10^{-1}$ and $\varepsilon_{F}=3.5 \times 10^{-1}$ is assumed, respectively. The initial state for agent $x_{i}(0)$ and target value $x_{i}^{*}$ were chosen to be

$$
\begin{aligned}
& x_{l}(0)=\left(\begin{array}{c}
-4 \\
-3 \\
-3 \\
2
\end{array}\right), \quad x_{2}(0)=\left(\begin{array}{c}
0 \\
3 \\
3 \\
-2
\end{array}\right), \quad x_{3}(0)=\left(\begin{array}{l}
3 \\
6 \\
1 \\
1
\end{array}\right) \\
& x_{l}^{*}=\left(\begin{array}{l}
0 \\
0 \\
0 \\
0
\end{array}\right), \quad x_{2}^{*}=\left(\begin{array}{c}
-1 \\
0 \\
-1 \\
0
\end{array}\right), \quad x_{3}^{*}=\left(\begin{array}{c}
1 \\
0 \\
-1 \\
0
\end{array}\right)
\end{aligned}
$$

as shown in the image in Fig. 2. Additionally, the weighting parameters $Q, R_{K}, R_{F}$ were set as $Q=1.0, R_{K}=2.0 \times 10^{-1}$, and $R_{F}=2.0 \times 10^{-1}$. Here, unknown parameter $\Delta K(t)$ and $\Delta F(t)$ is given as

$$
\begin{aligned}
& \Delta K=8.0 \times 10^{-1} \times \sqrt{\varepsilon_{K}} \times(1.0-\exp (-0.1 t)|\cos (10.0 \pi t)|) \times\left(\begin{array}{llll}
1 & 1 & 1 & 1 \\
1 & 1 & 1 & 1
\end{array}\right) \\
& \Delta F=8.0 \times 10^{-1} \times \sqrt{\varepsilon_{K}} \times(1.0-\exp (-0.1 t)|\cos (10.0 \pi t)|) \times\left(\begin{array}{llll}
1 & 1 & 1 & 1 \\
1 & 1 & 1 & 1
\end{array}\right)
\end{aligned}
$$



Fig. 2 Trajectories for each agent in the state space 
By applying Theorem 2 and solving the constrained convex optimization problem Eq. (36), there are the following values for the LMI solution:

$$
\begin{gathered}
S=\left(\begin{array}{cccc}
2.6795 \times 10^{-1} & -1.0585 \times 10^{-1} & -2.7372 \times 10^{-3} & -2.9836 \times 10^{-2} \\
* & 4.4751 \times 10^{-1} & 2.3603 \times 10^{-2} & 1.1958 \times 10^{-2} \\
* & * & 3.5287 \times 10^{-1} & -1.5169 \times 10^{-1} \\
* & * & * & 3.4045 \times 10^{-1}
\end{array}\right) \\
W_{K}=\left(\begin{array}{cccc}
2.3088 \times 10^{-1} & -4.5315 & 2.9135 \times 10^{-3} & 1.2169 \times 10^{-2} \\
8.8730 \times 10^{-4} & -8.2894 \times 10^{-3} & 2.3342 \times 10^{-3} & -4.4138
\end{array}\right) \\
W_{F}=\left(\begin{array}{cccc}
-1.1693 \times 10^{-9} & 6.4972 \times 10^{-9} & -4.4497 \times 10^{-10} & 7.0950 \times 10^{-9} \\
-2.3135 \times 10^{-10} & 5.8497 \times 10^{-9} & -6.6781 \times 10^{-10} & -6.1563 \times 10^{-8}
\end{array}\right) \\
\gamma=6.1981 \times 10^{-1}, \delta=1.3859, \zeta=5.0597, \eta=6.8027, \sigma=3.5265 \times 10^{2}
\end{gathered}
$$

Therefore, the feedback gain matrix and the consensus can be calculated as

$$
\begin{gathered}
K=\left(\begin{array}{cccc}
-4.2765 & -1.1199 \times 10^{1} & 9.2459 \times 10^{-1} & 4.6627 \times 10^{-1} \\
-1.7296 \times 10^{-1} & 3.7704 \times 10^{-1} & -7.0206 & -1.6257 \times 10^{1}
\end{array}\right) \\
F=\left(\begin{array}{cccc}
4.1277 \times 10^{-9} & 1.4406 \times 10^{-8} & 8.2922 \times 10^{-9} & 2.4390 \times 10^{-8} \\
-2.9470 \times 10^{-8} & 1.9930 \times 10^{-8} & -1.0160 \times 10^{-7} & -2.2850 \times 10^{-7}
\end{array}\right)
\end{gathered}
$$

On the other hand, the gain matrices for the conventional consensus controller can be obtained as

$$
\begin{aligned}
& K=\left(\begin{array}{cccc}
-1.2613 & -7.7305 \times 10^{-1} & 1.1617 \times 10^{-5} & 6.6800 \times 10^{-6} \\
1.6130 \times 10^{-5} & 4.7165 \times 10^{-6} & -1.2615 & -7.7315 \times 10^{-1}
\end{array}\right) \\
& F=\left(\begin{array}{cccc}
2.0593 \times 10^{-4} & 7.9111 \times 10^{-6} & 4.2830 \times 10^{-6} & -9.4814 \times 10^{-6} \\
4.7420 \times 10^{-5} & 1.7697 \times 10^{-6} & 2.3861 \times 10^{-6} & -1.5674 \times 10^{-6}
\end{array}\right)
\end{aligned}
$$

Note that the gain matrices of Eq. (47) and (48) in the conventional controller can be designed by solving LMI of Eq. (A.1) in Appendix and Theorem A.1, and it shows an LMI-based design method of the conventional consensus controller. The conventional controller is designed by considering the stabilization of the closed-loop system of Eq. (A.2).

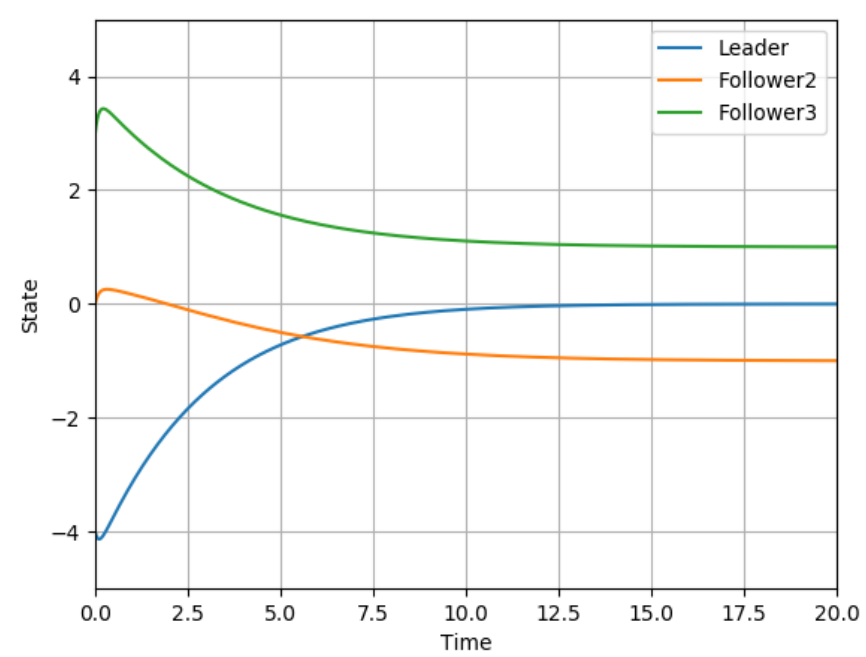

Fig. 3 Time histories of the $x$-position

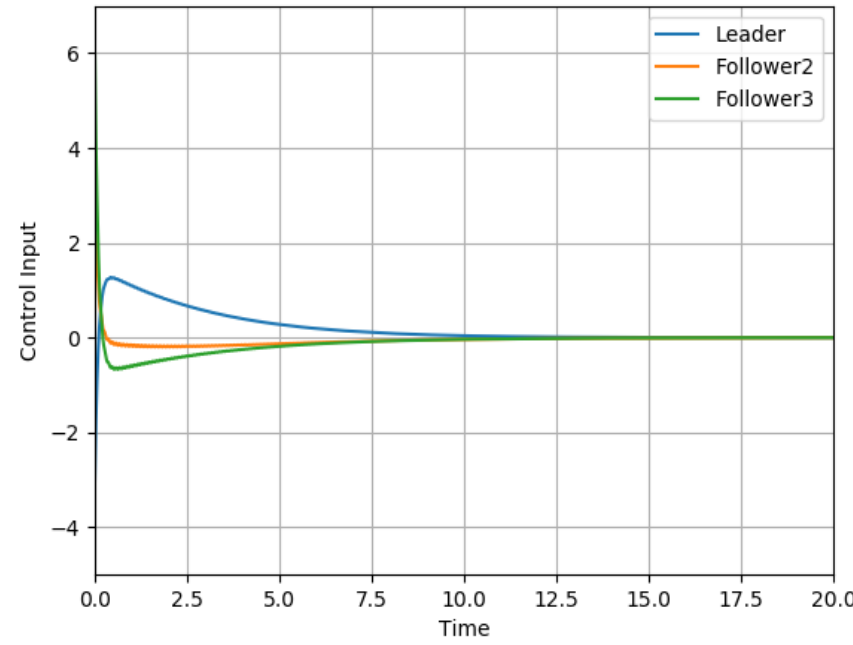

Fig. 4 Time histories of the control input for $x$-direction

The results of the simulation of this example were depicted in Figs. (3)-(10). Fig. 3 (resp. Fig. 5) shows the time histories of the $x$-position (resp. $y$-position), and Fig. 4 (resp. Fig. 6) presents those of the control input for $x$-direction(resp. $y$-position). In these figures, the transient time-response, the manipulated control input, and the actual control input generated 
by the proposed consensus controller, i.e. Figs. (3)-(6) are the results for the proposed consensus controller which is based on a guaranteed cost control strategy. Additionally, the figures (Figs. (7)-(10) show time histories of the state and the control input which were generated by the conventional consensus controller. Fig. 7 and Fig. 8 (resp. Fig. 9 and Fig. 10) represent the time histories of the $x$-position and one of the control input for $x$-direction (resp. the time histories of the $y$-position and one of the control input for $y$-direction), respectively.

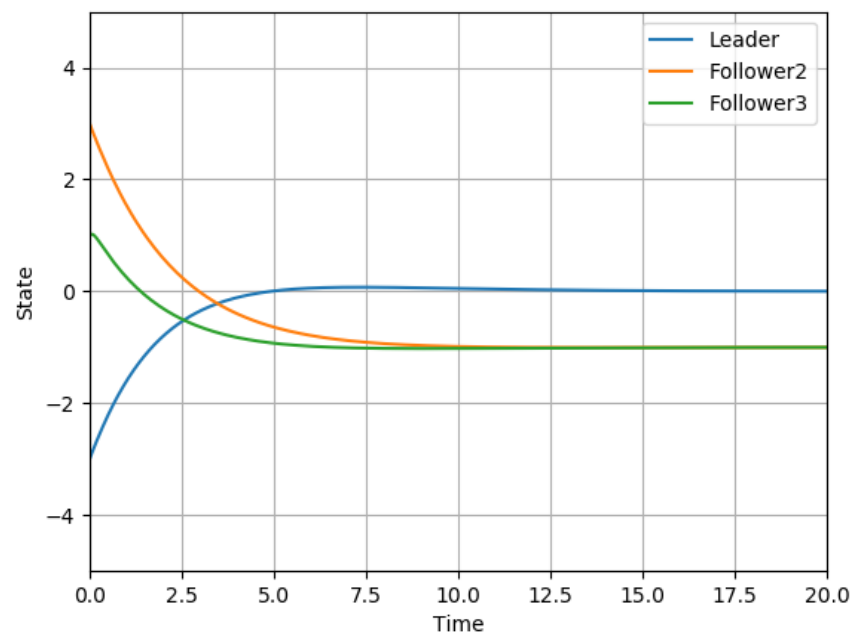

Fig. 5 Time histories of the $y$-position

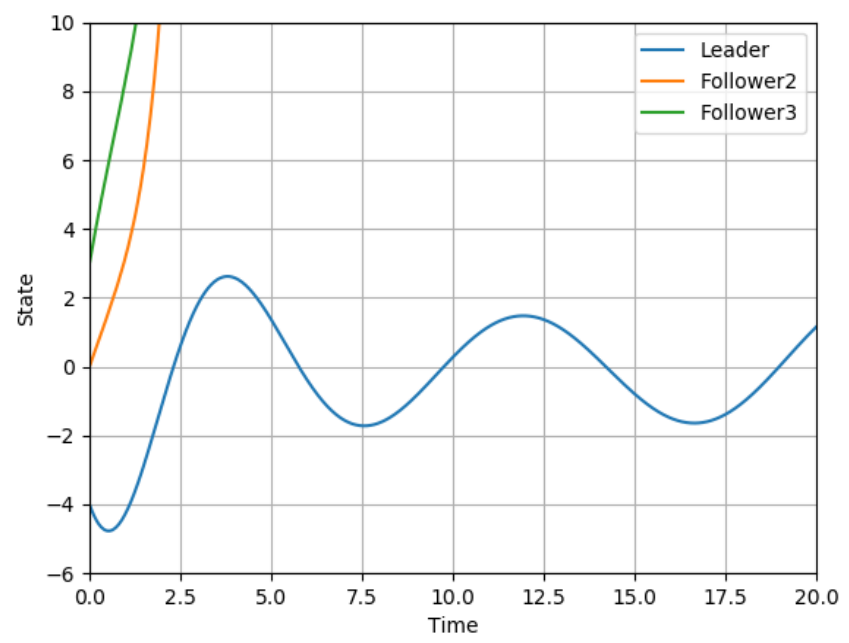

Fig. 7 Transient time-response of the $x$-position

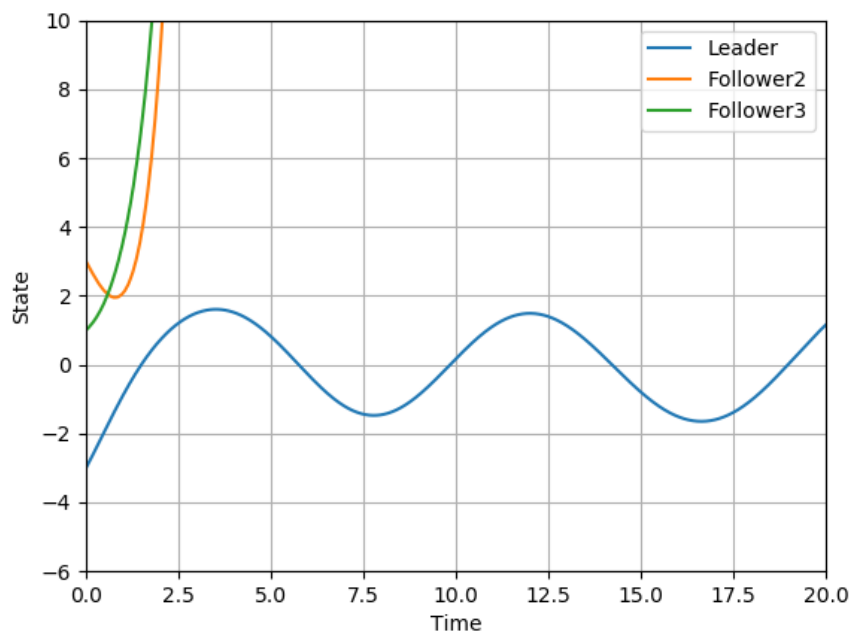

Fig. 9 Transient time-response of the $y$-position

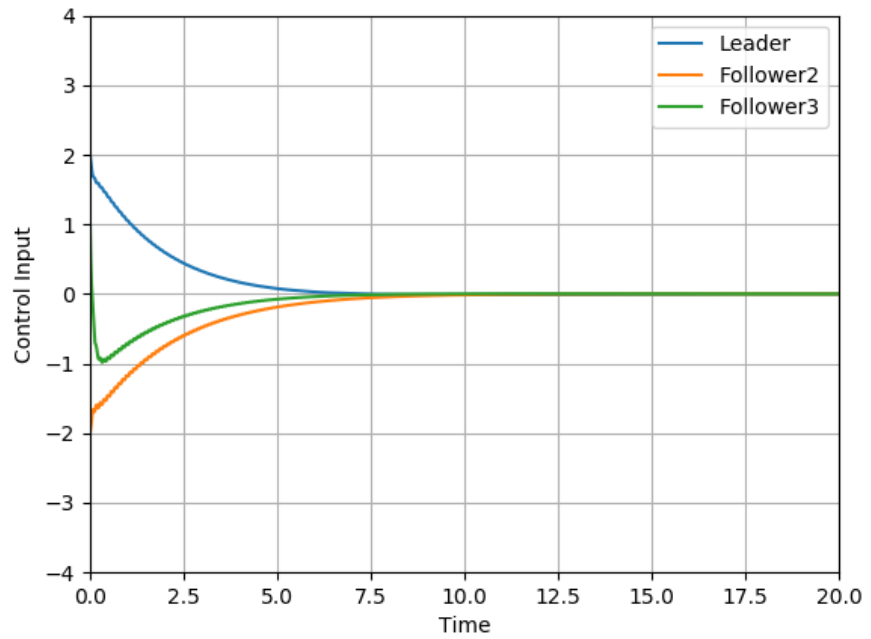

Fig. 6 Time histories of the control input for $y$-direction

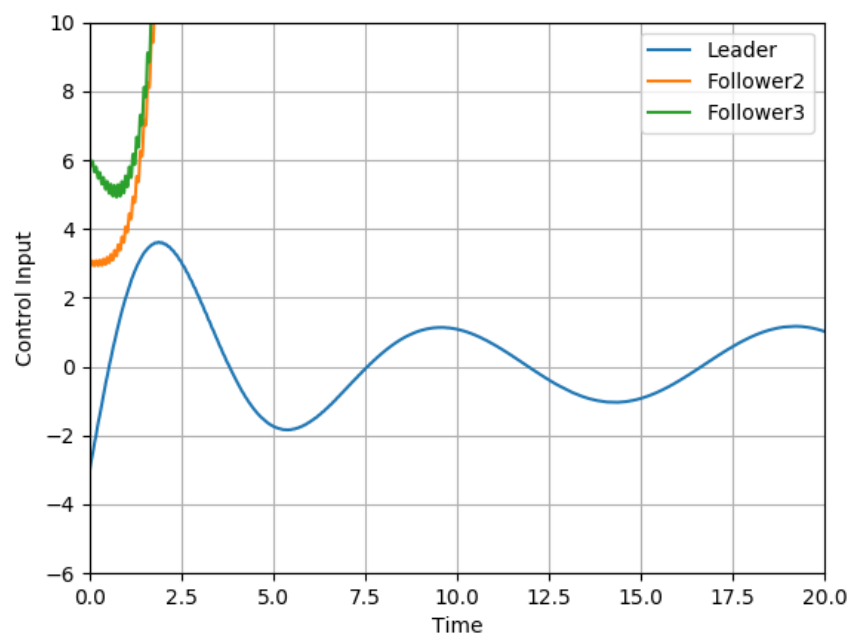

Fig. 8 Time histories of the control input $x$-direction

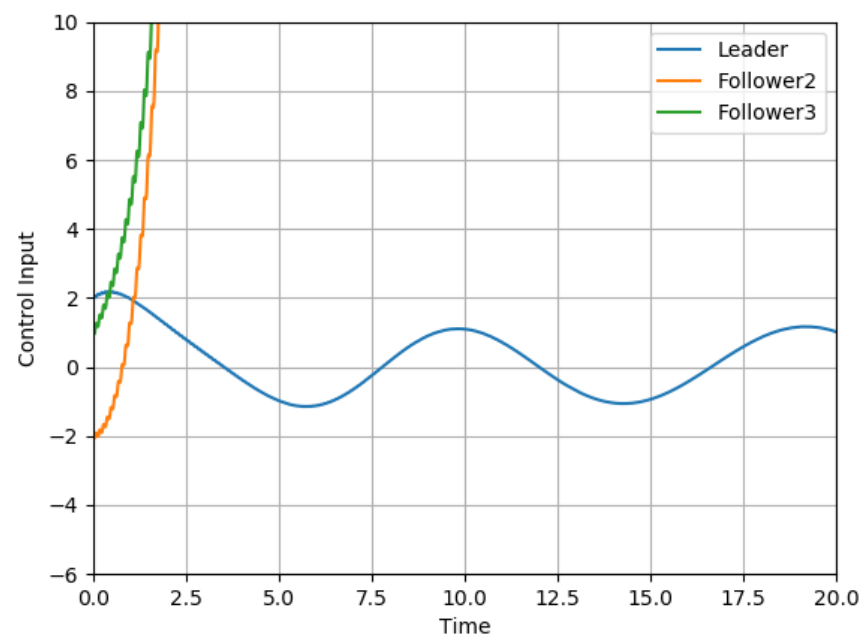

Fig. 10 Time histories of the control input $y$-direction 
From Figs. (2)-(10), the proposed non-fragile controller which was found stabilizes the uncertain linear system Eq. (5) under the control gain perturbation Eq. (41). Furthermore, the proposed non-fragile control strategy can achieve the consensus for multi-agent systems despite control gain perturbations. On the other hand, the conventional controller was found cannot achieve consensus because of the controller gain perturbations are ignored in the controller design stage. Therefore, the effectiveness of the proposed non-fragile stabilizing controller is shown.

\section{Conclusions}

This study had dealt with a design problem of a non-fragile guaranteed cost controller achieving consensus for MASs with leader-follower structures. sufficient conditions had been shown for the existence of the proposed non-fragile guaranteed controller can be reduced to the solvability of LMIs. Furthermore, the optimal non-fragile guaranteed cost controller, which minimizes the upper bound on a given quadratic cost function had been presented. Finally, simple numerical examples were given to illustrate the effectiveness of the proposed formation control system. The simulation result had shown that the closed-loop system was well stabilized despite control gain variations. Besides, the consensus for MASs can be achieved by the proposed non-fragile controller. Additionally, the proposed optimal guaranteed cost controller which had been shown can be designed by solving the constrained convex optimization problem. Namely, the proposed nonfragile controller can easily be obtained by using software such as MATLAB's LMI Control Toolbox and Scilab's LMITOOL.

It is worth pointing out that this paper didn't take into account the time delay that occurred when control was performed via a network. Therefore, there is room for expansion to the control system for systems with delays. Furthermore, future research subjects include some extensions of the proposed design approach to a broad class of systems such as discrete-time systems and output feedback systems. Additionally, the proposed controller synthesis will be extented to formation control for MASs consisting of more general agent's dynamics.

\section{Conflicts of Interest}

The authors declare no conflict of interest.

\section{Appendix}

In this appendix, an LMI-based design method of the conventional consensus controller which is showed was obtained by ignoring control gain perturbations. Namely, for $i$-th agent of Eq. (3), the following control input is considered:

$$
\begin{aligned}
u_{i}(t) & \triangleq u_{K, i}(t)+u_{F, i}(t) \\
& =K x_{i}(t)+F \sum_{j \in N_{i}}\left(x_{i}(t)-x_{j}(t)\right)
\end{aligned}
$$

Thus, the closed-loop system can be described as

$$
\begin{aligned}
\frac{d}{d t} x(t) & =A_{t} x(t)+B_{t}\left(K_{t} x(t)+F_{t} x(t)\right) \\
& =\left(\left(\begin{array}{ccc}
A & 0 & 0 \\
* & A & 0 \\
* & * & A
\end{array}\right)+\left(\begin{array}{ccc}
B & 0 & 0 \\
* & B & 0 \\
* & * & B
\end{array}\right)\left(\begin{array}{ccc}
K & 0 & 0 \\
-F & K+2 F & -F \\
-F & -F & K+2 F
\end{array}\right)\right)\left(\begin{array}{l}
x_{l}(t) \\
x_{2}(t) \\
x_{3}(t)
\end{array}\right) \\
& =\left(\begin{array}{ccc}
A_{K} & 0 & 0 \\
-B F & A_{F} & -B F \\
-B F & -B F & A_{F}
\end{array}\right)\left(\begin{array}{c}
x_{l}(t) \\
x_{2}(t) \\
x_{3}(t)
\end{array}\right)
\end{aligned}
$$

and the following LMI-based design method of the conventional consensus controller can be developed: 
Theorem A-1: Consider the overall system of Eq. (5) and the control input of Eq. (A.1).

If there exists a symmetric positive definite matrix $S>0$, matrices $W_{K}$ and $W_{F}$ will satisfy the following LMI condition:

$$
\left(\begin{array}{ccc}
H e\left\{A S+B W_{K}\right\} & -\left(B W_{F}\right)^{T} & -\left(B W_{F}\right)^{T} \\
* & H e\left\{A S+B W_{K}+2 B W_{F}\right\} & -H e\left\{B W_{F}\right\} \\
* & * & H e\left\{A S+B W_{K}+2 B W_{F}\right\}
\end{array}\right)<0
$$

then the closed-loop system of Eq. (A.2) with gain matrices $K=W_{K} S^{-1}$ and $F=W_{F} S^{-1}$ are asymptotically stable.

Proof: The result of Theorem A.1 can easily be obtained by using a similar way to the proof of Theorem 1 .

\section{References}

[1] K. Zhou, J. C. Doyle, and K. Glover, Robust and optimal control, Prentice Hall, 1996.

[2] K. Zhou, Essentials of robust control, Prentice Hall, 1998.

[3] B. R. Barmish, "Stabilization of uncertain systems via linear control," IEEE Transaction on Automat Control, vol. 28, no. 8, pp. 848-850, 1983.

[4] I. R. Petersen and C. V. Hollot, "A Riccati equation approach to the stabilization of uncertain linear systems," Automatica, vol. 22, no. 4, pp. 397-411, 1986.

[5] P. P. Khargonekar, I. R. Petersen, and K. Zhou, "Robust stabilization of uncertain linear systems: quadratic stabilizability and control theory," IEEE Transaction on Automatic Contrtol, vol. 35, no. 3, pp. 356-361, 1990.

[6] S. S. L. Chang and T. K. C. Peng, "Adaptive guaranteed cost control of systems with uncertain parameters," IEEE Transaction on Automatic Control, vol. 17, no. 4, pp. 474-483, 1972.

[7] I. R. Petersen and D. C. McFarlane, "Optimal guaranteed cost control and filtering for uncertain linear systems," IEEE Transaction on Automatic Control, vol. 39, no. 9, pp. 1971-1977, 1994.

[8] L. Yu and J. Chu, "Optimal guaranteed cost control of linear systems: LMI approach,” Proc. 14th IFAC World Congress, Beijing, China 1999, pp. 541-546.

[9] L. Yu and J. Chu, “An LMI approach to guaranteed cost control of linear uncertain time delay systems,” Automatica, vol. 35, no. 6, pp. 1155-1159, 1999.

[10] L. H. Keel and S. P. Bhattacharyya, "Robust, fragile, or optimal?,” IEEE Transaction on Automatic Control, vol. 42, no. 8, pp. 1098-1105, 1997.

[11] P. Dorato, "Non-fragile controller design: an overview," Proc. American Control Conference, Philadelphia, Pennsylvania, USA, pp. 2829-2831, 1998.

[12] D. Famularo, P. Dorato, C. T. Abdallah, W. M. Haddad, and A. Jadbabaie, "Robust non-fragile LQ controllers : the static state feedback case," Interational Journal of Control, vol. 73, no. 2, pp. 159-165, 2000.

[13] H. Mukaidani, Y. Takato, Y. Tanaka, and K. Mizukami, "The guaranteed cost control for uncertain large-scale interconnected systems," Proc. the 15th IFAC World Congress, pp. 265-270, 2002.

[14] H. Oya, K. Hagino, and H. Mukaidani, "Robust non-fragile controllers for uncertain linear continuous-time systems," Proc. the 31st Annual Conference of IEEE Industrial Society (IECON2005), Raleigh, NC, USA, pp. 1-6, 2005.

[15] D. D. Siljak, Decentralized control of complex systems, Courier Corporation, 1991.

[16] Z. Gong, "Decentralized robust control of uncertain interconnected systems with prescribed degree of exponential convergence," IEEE Transaction on Automatic Control, vol. 40, no. 4, pp. 704-707, 1995.

[17] S. Nagai, H. Oya, T. Kubo, and T. Matsuki, "Synthesis of decentralized variable gain robust controllers for a class of large-scale interconnected systems with mismatched uncertainties,” International Journal of System Science, vol. 48, no. 8, pp. 1616-1623, 2017.

[18] H. Mukaidani, Y. Tanaka, and K. Mizukami, "Guaranteed cost control for large-scale systems under control gain perturbations,” Electrical Engineering in Japan, vol. 146, no. 4, pp. 43-57, 2004. 
[19] R. Olfati-Saber and R. M. Murray, "Consensus problems in networks of agents with switching topology and timedelays," IEEE Transaction on Automatic Control, vol. 49, no. 9, pp. 1520-1533, 2004.

[20] G. Xie and L. Wang, "Consensus control for a class of networks of dynamic agents: fixed topology,” Proc. the 44th IEEE Conference on Decision and Control, and the European Control Conference 2005, Seville, SPAIN, 2005, pp. 96101.

[21] Y. Zhang and Y. P. Tian, "Consentability and protocol design of multiagent systems with stochastic switching topology," Automatica, vol. 45, no. 5, pp. 1195-1201, 2009.

[22] G. Zhai, S. Okuno, J. Imae, and T. Kobayashi, "A matrix inequality based design method for consensus problems in multi-agent aystems," Internationl Journal of Application Mathematics and Computer Science, vol. 19, no. 4, pp. 639646, 2009.

[23] S. Ito, K. Miyakoshi, H. Oya, Y. Hoshi, and S. Nagai, "Consensus via adaptive gain controllers considering relative distances for multi-agent systems," Advcances in Technology Innovation, vol. 4, no. 4, pp. 234-246, August 2019.

[24] S. Boyd, L. El Ghaoui, E. Feron, and V. Balakrishnan, Linear matrix inequalities in system and control theory, SIAM Studies in Applied Mathmatics, 1994.

[25] H. Oya, K. Hagino and H. Mukaidani, "Guaranteed. cost control for uncertain. linear continuous-time. systems. under conrtol gain perturbations,” (in Japanese) Trans. of JSME(C), vol. 72, no. 713, pp. 92-101, 2006.

Copyright $(\mathrm{C}$ by the authors. Licensee TAETI, Taiwan. This article is an open access article distributed under the terms and conditions of the Creative Commons Attribution (CC BY-NC) license (https://creativecommons.org/licenses/by-nc/4.0/). 